\title{
Initial TCV Operation with a Baffled Divertor
}

\author{
H. Reimerdes ${ }^{1}$, B.P. Duval ${ }^{1}$, H. Elaian ${ }^{1}$, A. Fasoli ${ }^{1}$, O. Février ${ }^{1}$, C. Theiler ${ }^{1}$, F. Bagnato ${ }^{1}$, \\ M. Baquero-Ruiz ${ }^{1}$, P. Blanchard ${ }^{1}$, D. Brida ${ }^{2}$, C. Colandrea $^{1}$, H. De Oliveira ${ }^{1}$, \\ D. Galassi ${ }^{1}$, S. Gorno ${ }^{1}$, S. Henderson ${ }^{3}$, M. Komm ${ }^{5}$, B. Linehan ${ }^{4}$, L. Martinelli ${ }^{1}$, \\ R. Maurizio ${ }^{1}$, J.-M. Moret ${ }^{1}$, A. Perek ${ }^{6}$, H. Raj ${ }^{1}$, U. Sheikh ${ }^{1}$, D. Testa ${ }^{1}$, M. Toussaint ${ }^{1}$, \\ C.K. Tsui ${ }^{7}$, M. Wensing ${ }^{1}$, the TCV Team ${ }^{a}$ and the EUROfusion MST1 Team ${ }^{b}$
}

${ }^{1}$ Ecole Polytechnique Fédérale de Lausanne (EPFL), Swiss Plasma Center (SPC), Lausanne, Switzerland
${ }^{2}$ Max-Planck-Institut für Plasmaphysik, Garching bei München, Germany
${ }^{3}$ UKAEA, Culham Science Centre, Abingdon, UK
${ }^{4}$ Plasma Science and Fusion Center, Massachusetts Institute of Technology, Cambridge, USA
${ }^{5}$ Institute of Plasma Physics of the Czech Academy of Sciences, Prague, Czech Republic
${ }^{6}$ DIFFER, Dutch Institute for Fundamental Energy Research, Eindhoven, Netherlands
${ }^{7}$ Center for Energy Research, University of California San Diego (UCSD), La Jolla, USA
E-mail: holger.reimerdes@epfl.ch

Received 9 September 2020, revised 26 November 2020

Accepted for publication 8 Dember 2020

Published 11 January 2021

\begin{abstract}
The TCV tokamak is in the midst of an upgrade to further its capability to investigate conventional and alternative divertor configurations. To that end, modular and removable gas baffles have been installed to decrease the coupling between the divertor and the plasma core. The baffles primarily seek to suppress the transit of recycling neutrals to closed flux surfaces. A first experimental campaign with the gas baffles has shown that the baffled divertor remains compatible with a wide range of configurations including Snowflake and Super-X divertors. Plasma density ramp experiments reveal an increase of the neutral pressure in the divertor by up to a factor $\mathrm{x} 5$ compared to the unbaffled divertor and thereby qualitatively confirm simulations with the SOLPS-ITER code that were used to guide the baffle design. Together with a range of new and upgraded divertor diagnostics, the baffled TCV divertor is now used to validate divertor models for ITER and next step devices with particular emphasis on geometric variations.
\end{abstract}

Keywords: TCV tokamak, plasma exhaust, divertor

\section{Introduction}

The exhaust of heat and particles remains a critical issue for a fusion power plant based on the tokamak concept and extrapolations from today's devices remain subject to considerable uncertainties [1]. Current research seeks to improve the exhaust performance to assure feasibility and improve economical attractiveness of reactor concepts as well as test our confidence in such predictions [2,3].

The Tokamak à Configuration Variable (TCV) is a flexible, midsized tokamak [4,5] with a major radius $R_{0} \approx 0.88 \mathrm{~m}$ and a magnetic field $B_{\mathrm{T}} \approx 1.43 \mathrm{~T}$. With its 16 independently powered poloidal field coils, it is uniquely suited to investigate the effect of divertor geometry by being able to test a range of concepts within the same device without hardware modifications. An open divertor, together with a low opacity of the divertor to neutrals, generates a strong coupling between divertor and core that can limit highly dissipative divertor regimes.

As part of ongoing enhancements of the TCV tokamak [6], this coupling is reduced by inserting gas baffles on the highfield side (HFS) and low-field side (LFS) of the divertor entrance, effectly dividing the vacuum vessel into main and divertor chambers [7]. The baffles primarily seek to reduce the

\footnotetext{
${ }^{a}$ See the author list of S. Coda et al 2019 Nucl. Fusion 59112023

b See the author list of B. Labit et al 2019 Nucl. Fusion 59086020
} 
transit probability of recycling neutrals from the divertor towards the core. Their design was optimised, using the SOLPS-ITER code, to maximise the neutral compression in the divertor [8]. The optimum baffle dimensions were found to be a trade-off between restricting neutral conductance from divertor to main chamber and recycling off the top of the baffles themselves. In addition to improving TCV's divertor performance, the option of inserting baffles is a unique opportunity to test the models used to design the ITER divertor [9].

To maintain the flexibility of the TCV facility, the baffles were designed as extended graphite tiles that replace existing wall protection tiles during a manned entry [10]. The baffles are removable with their installation foreseen for dedicated experimental campaigns that may be interleaved with campaigns with TCV in its original configuration. The baffle tiles were shaped to avoid excessive surface heating during routine operation with a mechanical mount to the vacuum vessel able to withstand the electromagnetic forces expected during disruptions.

This letter seeks to inform the fusion community of the first major results of TCV's new baffled divertor configuration. Section 2 shows that the new configuration remains compatible with a wide range of plasma shapes and operational regimes. Section 3 reports on experiments that demonstrate the functionality of the baffled divertor. These experiments are used to conclude in Section 4 that the design objectives are met, providing a new physics testbed for the research of highly dissipative conventional and alternative divertors.

\section{TCV operation with gas baffles}

The HFS baffle was inserted in early 2019 and operated for a brief experimental campaign before the installation of the LFS baffle. The HFS baffle installation was accompanied by an ex-vessel cleaning of all TCV's graphite protection tile surfaces. Abrasive blasting with cubic boron nitride grains physically removed a thin surface layer restoring a pristine graphite surface. The subsequent LFS baffle installation was accompanied by additional port protection tiles that shadow the lower lateral ports from direct plasma exposure along scrape-off layer magnetic field lines. The port protection tiles are a prerequisite for any magnetic configuration with a strike point on the outer wall of the new divertor chamber. They are independent of the baffles and increase the configurational flexibility for both baffled and unbaffled TCV. The installation of the baffles occurred together with the relocation of one of three gas valves (valve \#2) from the floor to the ceiling of the TCV vessel permitting main chamber fuelling or seeding in addition, or as an alternative, to divertor fuelling or seeding. The new divertor components are shown in Fig. 1.

TCV operation following the installation of both baffles proceded without problems and limited together with diverted configurations were promptly obtained. The diverted configuration, Fig. 1(b), employed a newly developed startup that keeps the plasma limited on the inboard wall during the formation of an in-vessel X-point. The divertor is then activated by displacing the plasma outwards, thereby, avoiding significant heat loads on the baffle tiles at all times.

Low density operation in L-mode remains limited by runaway electron generation. Stationary $\mathrm{H}$-mode regimes with regular ELMs were readily established over a wide range of plasma densities and currents. The H-mode power threshold was found to be similar, or lower, than without the baffles.

a)
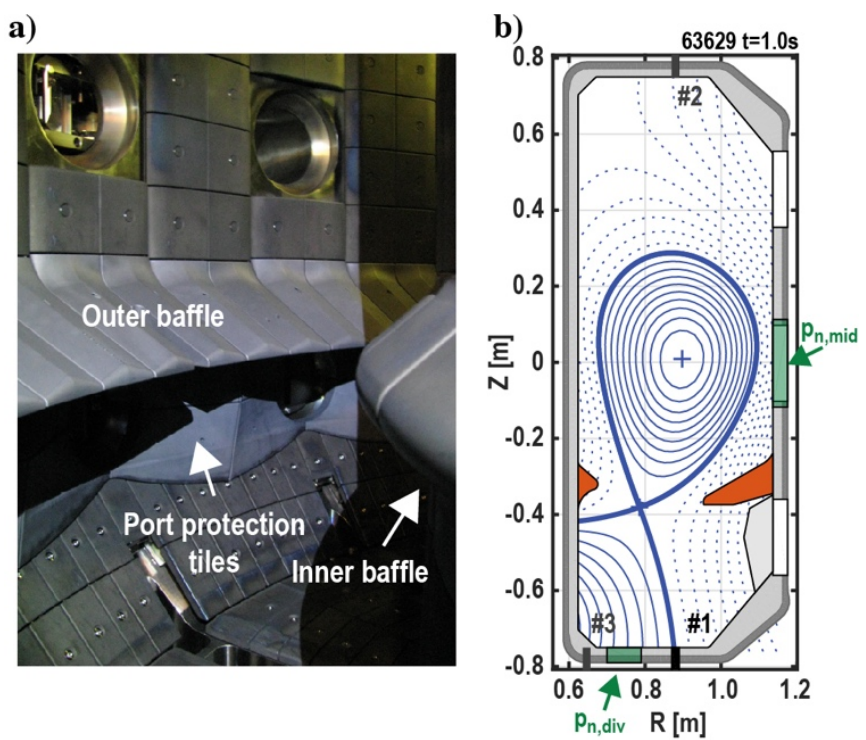

Figure 1. (a) Interior of the TCV vacuum vessel and (b) poloidal cross section of a standard single-null configuration in the baffled $T C V$ vessel. Highlighted are the HFS and LFS baffles (orange), gas valves (black) and ports equipped with baratrons (green).

As the divertor baffles installation in TCV primarily aims at the study of alternative divertor configurations, accessibility of a wide range of alternative divertors was demonstrated, Fig. 2. The realised configurations are accessible to heating with TCV's neutral beam injection (NBI) and electron cyclotron heating $(\mathrm{ECH})$ systems. Limited configurations with an elongation up to 2.0 remain feasible within the main chamber.
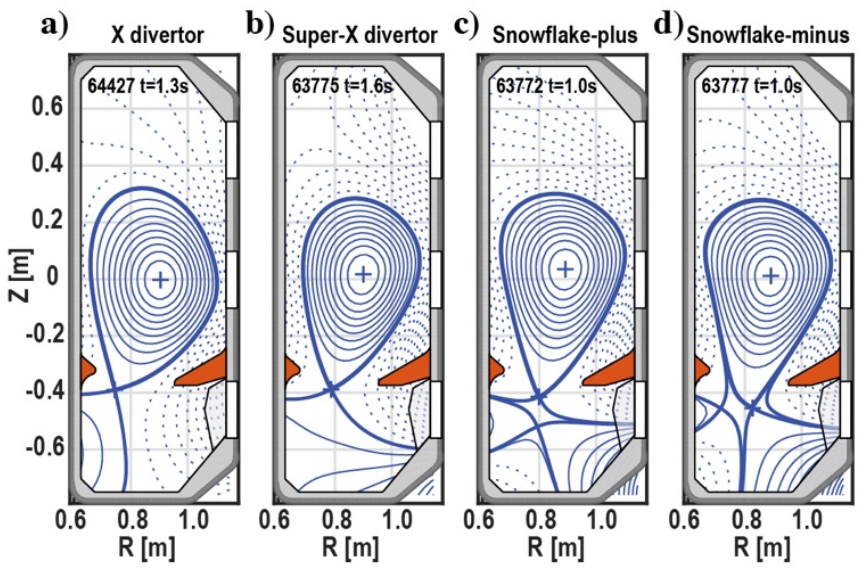

Figure 2. (a) X, (b) Super-X, (c) Snowflake-plus and (d) Snowflakeminus configurations realised in TCV with a baffled divertor.

TCV operated in the baffled configuration for the remainder of the 2019 experimental campaign. 

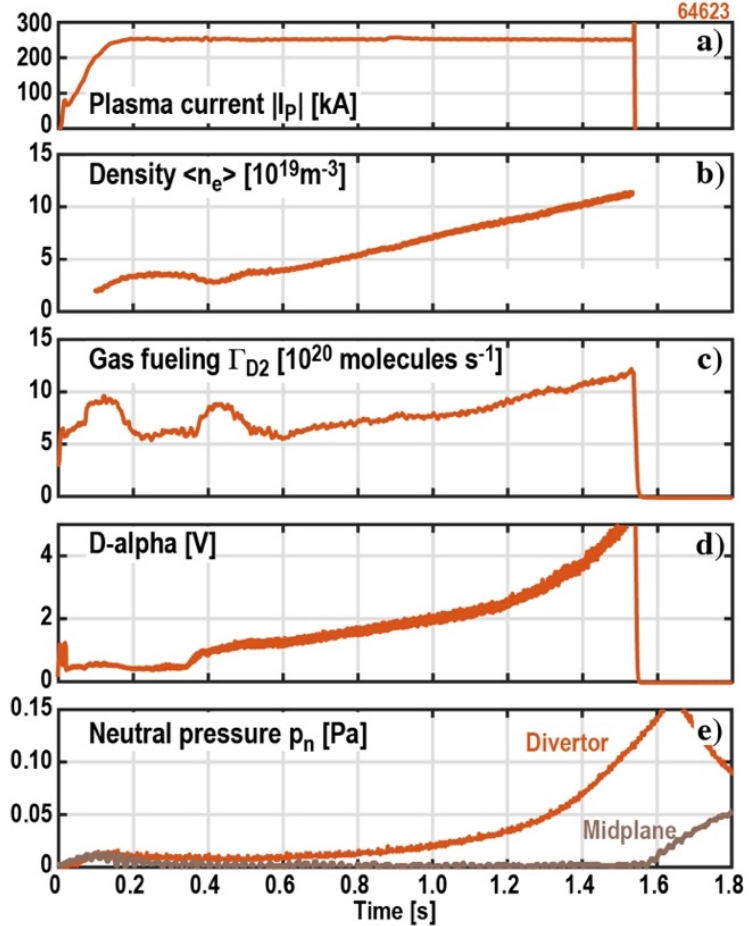

Figure 3. (a) Ohmically heated discharges in baffled single-null configurations, Fig. 1(b), with a (b) density ramp obtained by (c) divertor gas fuelling (using valve \#1). Shown are also (d) D-alpha emission and (e) neutral pressure measurements in the divertor and outboard midplane.

\section{Divertor performance}

The performance of the baffled TCV divertor has been assessed in Ohmically heated deuterium plasmas in a standard, single-null (SN), configuration, Fig. 1(b), where divertor gas fuelling (using valve \#1) leads to a feedback-controlled increase of the electron density up to a disruptive limit, Fig. 3. To remain in L-mode, the toroidal magnetic field is directed such that the ion grad B drift is from the X-point to the plasma core (i.e. out of the divertor and unfavourable for $\mathrm{H}$-mode access). A key indicator of the divertor performance is the neutral compression between the divertor and the main chamber, section 3.1. The neutral pressure is measured with baratron pressure gauges installed in a divertor port, located in the private flux region of the investigated SN configuration, and an outboard midplane port, Fig. 1(b). The strike points are positioned sufficiently far from the divertor baratron port entrance to avoid any local increase of the neutral pressure in the vicinity of the strike points. It is noted that the neutral conductance of the vacuum components and the response of the baratron introduces a characteristic time constant of the order of $100 \mathrm{~ms}$. The observed increase of the neutral pressure in the baffled divertor eases the access to detachment, section 3.2. The density limit can be, but not always, preceded by a jump in density, indicating a transition in the divertor regime, discussed in section 3.3.

\subsection{Neutral compression}

As the baffles were designed to maximise the neutral compression, the neutral pressure measurements in the baffled configuration, Fig. 3(e), are compared with measurements in an identically programmed discharge prior to the installation of the baffles, Fig. 4. The obtained magnetic configurations are highly reproducible with strike point locations agreeing to within a few millimetres. Measured neutral pressures and attained density limits, however, vary with wall-conditioning. Reported values are, therefore, only dependencies that cannot be explained by these variations.
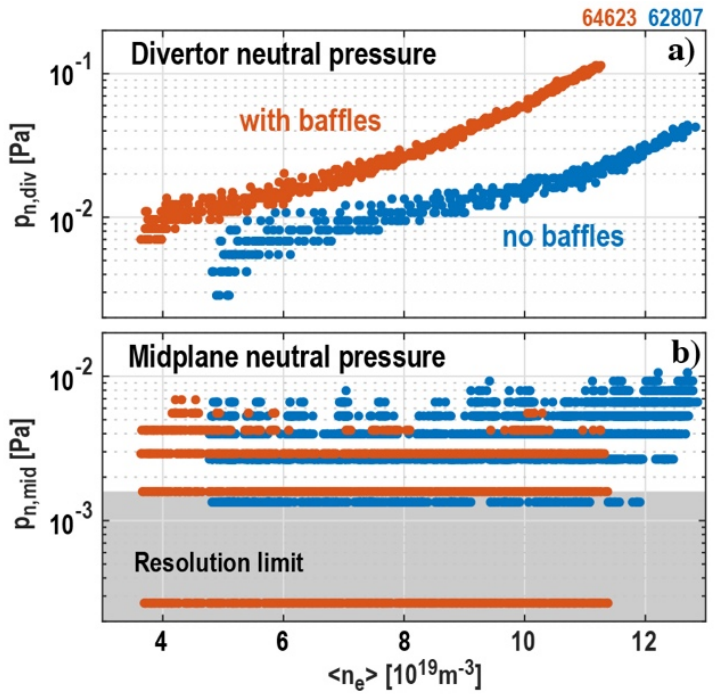

Figure 4. Baratron measurements of the neutral pressure in the (a) divertor and (b) main chamber in similar discharges with (orange) and without (blue) baffles.

Discharges with the baffles and divertor gas fuelling generally have a higher divertor neutral pressure, $p_{\mathrm{n} \text {,div }}$, for the same plasma density and typically reach a higher value of $p_{\text {n,div }}$ before plasma disruption, Fig. 4(a). The density limit is, however, similar or even slightly lower than for the unbaffled configuration. The midplane neutral pressure, $p_{n, \text { mid }}$, for baffled and unbaffled configurations is, unfortunately, close to the lower sensitivity limit of the installed baratron, Fig. 4(b). Measurements suggest that the baffles lower the midplane neutral pressure, but such a conclusion requires improved, future, diagnostics.

The observed increase of $p_{\mathrm{n} \text {,div }}$ with the baffles by a factor of 2-5 at the same plasma density and depending on density and device conditions is consistent with the SOLPS-ITER simulations used to guide the baffle design $[8,11]$ as well as subsequent SOLEDGE2D-Eirene simulations [12]. While SOLPS-ITER correctly predicts the magnitude of the increase, it tends to overestimate the absolute value of the neutral pressure by up to an order of magnitude. A similar overestimation of the divertor neutral pressure by an edge fluid code was already observed in SOLPS5.2 simulations of the small angle slot divertor in DIII-D [13]. The reasons for these discrepancies are not understood and remain subject of ongoing investigations with the role of particle drifts, neglected in all simulations mentioned above, potentially important. 


\subsection{Access to detachment}

High neutral compression is expected to increase plasma energy and momentum dissipation within the divertor while remaining below the core plasma density limit. Transferring energy and momentum from the plasma to neutrals or photon emission, a key requirement for detachment, is favourable for power exhaust as neutral particles and radiation will distribute energy deposition over a much greater surface area. Detachment is often characterised by a sufficiently low electron temperature in the proximity of the targets and a change in the scaling of the ion current at the divertor targets with density, leading to a 'roll-over' of the target current in density ramp experiments. Detachment, thereby, reduces physical sputtering and heat flux to the targets.

An estimate of the electron temperature in the divertor is obtained from spectral line emission of neutral or partially ionised species. As the electron energy required for line excitation and ionisation effectively bracket the electron temperature, the emission region is characteristic for an electron temperature range. Spectral imaging of the CIII line at $465 \mathrm{~nm}$ is regularly used to characterise the divertor plasma $[14,15]$ with the lower temperature limit being $\sim 10 \mathrm{eV}[16]$. For the same core density, the location of the lower CIII emission limit in the baffled divertor is closer to the X-point, Fig. 5, indicating that the electron temperature in the outer divertor has been, generally, reduced.

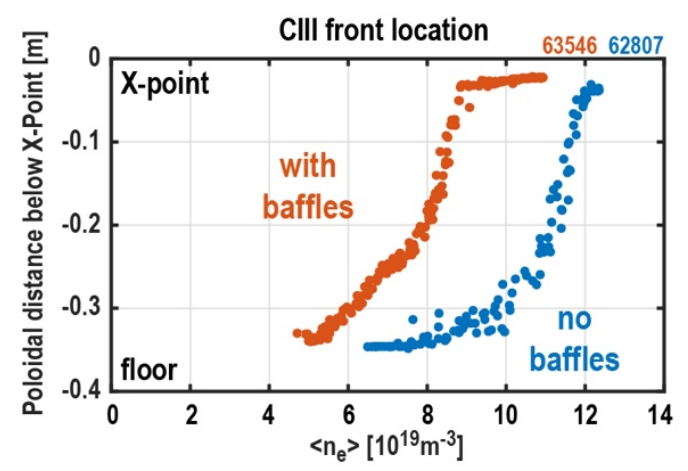

Figure 5. Dependence of the location of the CIII front with respect to the X-point on plasma density in similar discharges with (orange) and without (blue) baffles.

Target ion fluxes, measured by wall mounted Langmuir probes, confirm that the baffles facilitate access to detachment, Fig. 6. In discharges with baffles and divertor gas fuelling, the characteristic roll-over generally occurs at lower plasma densities. In the investigated Ohmic discharges the roll-over at the outer target, Fig. 6(a), can occur at densities sometimes $30 \%$ lower than for un-baffled discharges. There is, however, a significant variance among nominally identical discharges indicating an influence of wall-conditioning and pervasive impurity concentrations. In addition to the ion current roll-over at lower plasma density, discharges with the baffled divertor can also display a roll-over of the ion current at the inner target, Fig. 6(b), not observed for unbaffled TCV discharges, indicating a stronger coupling between the inner and outer divertor.
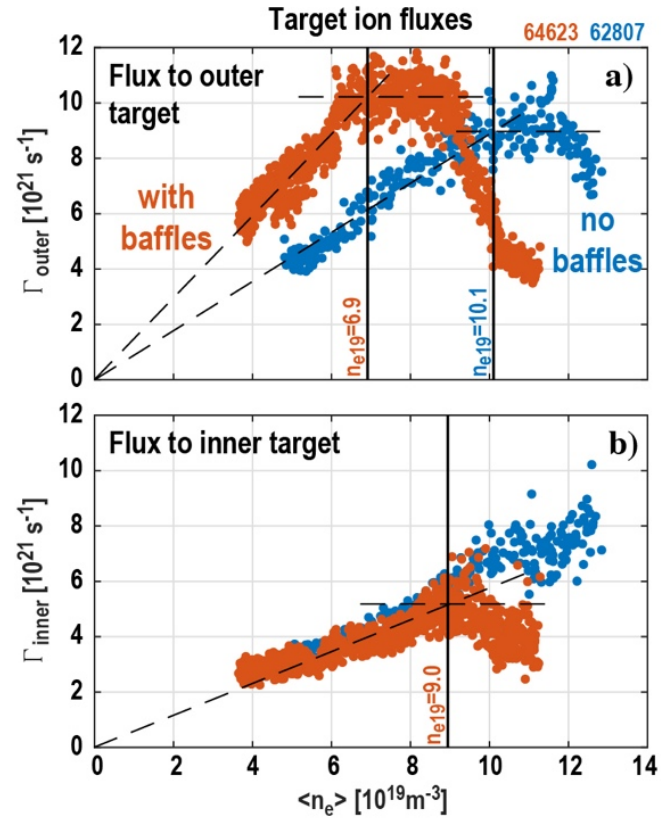

Figure 6. Ion flux on the (a) outer and (b) inner divertor targets measured with wall mounted Langmuir probes in similar discharges with (orange) and without (blue) baffles. Intersections of linear fits yield estimates of the density at the onset of the roll-over.

\subsection{Plasma operation in the detached regime}

The baffles decrease the density required for detachment onset with only a small effect on the density limit, thus broadening TCV's operational parameter space with a detached divertor.

The density limit is often preceded by a sudden, transient, increase of $n_{\mathrm{e}}$, Fig. 7(a), that was not observed for the unbaffled divertor. A decrease of the gas fuelling rate after the increase in $n_{\mathrm{e}}$, Fig. 7(b), indicates a transition to a divertor regime that is significantly less opaque to divertor gas fuelling. This transition is accompanied by an equally sudden increase of soft X-ray (SXR) emissivity, Fig. 7(c), and core carbon density. This transition is not observed immediately after boronisation, indicating a role of $\mathrm{C}$ impurities. It is hypothesised that ionisation of divertor neutrals, in combination with the $\mathrm{C}$ content of the divertor, can lead to a radiation-condensation instability that causes a rapid loss of the neutral opacity of the plasma in the vicinity of the X-point, also referred to as "plasma plugging", of the baffled divertor. 

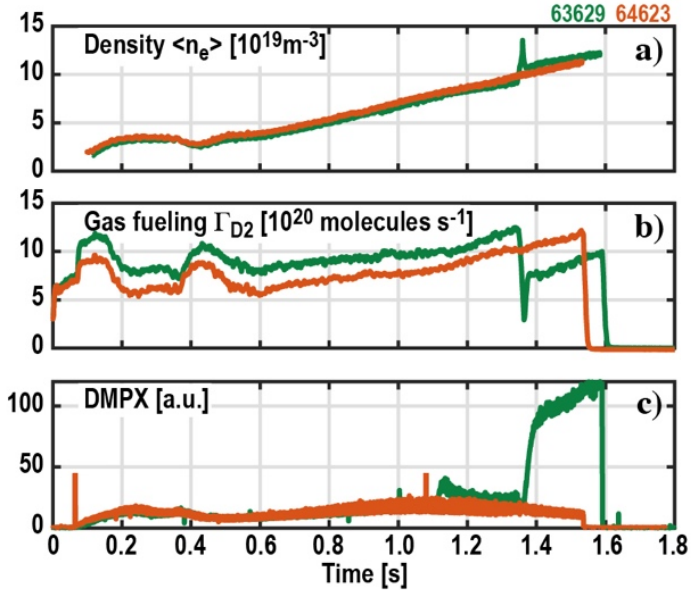

Figure 7. Identically programmed density ramp experiments, both in the baffled configuration, with (green) and without (orange) a sudden increase of the density (a). The increase coincides with changes in the (b) divertor gas-fuelling rate and (c) SXR emission measured with a central DMPX chord.

\section{Conclusions}

The TCV tokamak has recently been equipped with a set of gas baffles that create a closed divertor. Their installation has not adversely affected plasma operation and a wide range of plasma configurations were readily obtained. The baffles have, however, a profound effect on the divertor performance. In particular, they lead to an increase of the neutral pressure in the divertor by up to a factor of $\mathrm{x} 5$, broadly in line with modelling. This is accompanied by a decrease of the lineaveraged plasma density at onset of detachment. The baffled discharges also display an increased coupling between the inner and the outer divertor. The presented initial experiments confirm that the baffles have met their design objectives. They are able to test divertor models and are, consequently, now extensively used together with a range of new and upgraded divertor diagnostics $[7,8]$ in the investigation of conventional and alternative divertors. A comprehensive analysis of the first TCV campaign with a baffled divertor including the effect of the baffles on H-mode exhaust and impurity seeding experiments will be presented at the upcoming PSI conference [17].

\section{Acknowledgements}

This work has been carried out within the framework of the EUROfusion Consortium and has received funding from the Euratom research and training program 2014-2018 and 20192020 under grant agreement No. 633053. The views and opinions expressed herein do not necessarily reflect those of the European Commission. This work was supported in part by the Swiss National Science Foundation and the US Department of Energy under Award Number DE-SC0010529.

\section{References}

[1] Federici G. et al 2019 Nucl. Fusion 59066013

[2] Zohm H. et al 2020 "The EU Strategy for solving the DEMO Exhaust Problem", 31st Symposium on Fusion Technology, virtual edition, 2020, submitted to Fusion Eng. Des.
[3] Reimerdes H. et al 2020 Nucl. Fusion 60066030

[4] Hofmann F. et al 1994 Plasma Phys. Control. Fusion 36 B277

[5] Coda S. et al 2019 Nucl. Fusion 59112023

[6] Fasoli A. for the TCV Team 2015 Nucl. Fusion 55043006

[7] Reimerdes H. et al 2017 Nucl. Mater. Energy 121106

[8] Fasoli A. et al 2020 Nucl. Fusion 60016019

[9] Pitts R.A. et al 2019 Nucl. Mater. Energy 20100696

[10]Vaccaro D. et al 2019 Fus. Eng. Des. 1461543

[11]Wensing M. et al 2019 Plasma Phys. Control. Fusion 61 085029

[12] Galassi D. et al 2020 Plasma Phys. Control. Fusion 62115009

[13]Shafer M.W. et al 2019 Nucl. Mater Energy 19487

[14]Fenstermacher M.E. et al 1997 Phys. Plasmas 41761

[15]Harrison J.R. et al 2017 J. Nucl. Mater. 121071

[16]Smolders A. et al 2020 Plasma Phys. Control. Fusion 62 125006

[17]Février O., the TCV team and the MST1 team, "First results from the new baffled TCV divertor", 24th International Conference on Plasma Surface Interactions in Controlled Fusion Devices, Jeju, Korea, 2021, submitted to Nucl. Mater. Energy 